\title{
SURFACE GREEN'S FUNCTION OF A SOFT ELASTOMER WITH THE GRAVITY EFFECT
}

\author{
XIAODONG LiANG \\ Hefei University, Department of Architectural Engineering, Hefei, P.R. China \\ e-mail: lxd@hfuu.edu.cn \\ Junxiu Liu, Peibao Xu, Kai Li \\ Anhui Jianzhu University, Department of Civil Engineering, Hefei, P.R. China \\ e-mail: tju_liu@hotmail.com; peibaoxu@ahjzu.edu.cn; kli@ahjzu.edu.cn
}

\begin{abstract}
Gravity can play a tremendous effect on deformation of rubberlike materials and biological soft tissues. In this paper, considering the gravity effect, we proposed a surface Green's function of a soft elastomer based on the neo-Hookean model. The proposed surface Green's function is applied to analyze the elastic deformation of a soft elastomer subjected to uniform pressure. The surface normal displacement of the soft elastomer is calculated and the results show that gravity has a large impact on the surface deformation of the elastomer. Generally, the surface normal displacement decreases with the increasing gravitational force.
\end{abstract}

Keywords: surface Green's function, gravity, distributed pressure, soft materials, neo-Hookean model

\section{Introduction}

Numerous materials like rubbers, biological soft tissues and polymer gels can produce large deformations due to lower elastic modulus even though the surface of soft materials is subject to small forces. In the last decade, stemming from the elastic deformation, instability patterns in soft materials could be observed in experiments and elucidated theoretically (Tanaka et al., 1987; Dervaux and Amar, 2012; Kim et al., 2012; Zhu et al., 2010; Keplinger, 2012; Gent and Cho, 1999; Biot, 1963; Wang and Zhao, 2013). In attempt to generate and regulate a surface pattern accurately, the elastic deformation of soft materials shall be understood and clarified rigorously. The well-known Green's function for the conventional elastic half space has been well built and provides an important method for calculating the elastic deformation of soft materials. Furthermore, the surface Green's functions of soft solids were first put forward incorporating the influence of surface stress (He and Lim, 2006) and prestretches (He, 2008), respectively.

Recently, the effect of gravity on elastic deformation of soft solids has attracted much attention (Mora et al., 2014; Liang and Cai, 2015; Amar and Jia, 2013; Li et al., 2012). To evaluate the effect of gravity, an intrinsic length scale is often defined as $L_{0}=\mu /(\rho g)$, where $\mu$ is the shear modulus of the solid, $\rho$ is density, and $g$ is gravitational acceleration. When the characteristic length of a soft material is comparable or larger than the intrinsic length scale, gravity may play a significant role on the elastic deformation of the gel, elastomer and a soft biological tissue (Mora et al., 2014; Liang and Cai, 2015; Amar and Jia, 2013; Li et al., 2012). Motivated by the thought, the surface Green's function of a soft elastomer occupying the half space is derived in this paper considering the effect of gravity. As an example, the surface deformation resulted from uniform distributed pressure in a circular zone is investigated.

In this paper, we will mainly focus on the effect of gravity on the surface deformation of soft materials, by ignoring the effect of surface stress and prestretches, although they may 
have a large impact on the elastic deformation of a soft elastomer. The organization of this paper is as follows. In Section 2, following the methodology (He and Lim, 2006; He, 2008; Biot, 1965; Ogden, 1997), we firstly derive the surface Green's function of an elastomer occupying a half space incorporating the gravity effect based on the neo-Hookean constitutive model. In Section 3, we secondly investigate the surface elastic deformation of an elastomer subjected to uniform distributed pressure in a circular zone by using the derived Green's function. Finally, the conclusion is given in Section 4 .

\section{Derivation of surface Green's function of an elastomer occupying a half space with the gravity effect}

For a homogeneous, isotropic and incompressible solid elastomer, the surface Green's function of a half space has been deduced considering the surface stress (He and Lim, 2006) and prestretches (He, 2008). So far, the surface Green's function has not been available involving the gravity effect which may have a non-negligible influence on the elastic deformation of soft materials. Here, we will derive the surface Green's function of an elastomer occupying a half space taking into account gravity.

We first consider a homogeneous, elastically isotropic and incompressible elastomer occupying a half space. When the elastomer stays in an initial unstressed state with a flat surface, we mark a material particle in the elastomer with coordinate $X_{K}$ as the initial state, and use $x_{i}$ to indicate the spatial position coordinate as an intermediate state.

A Cartesian coordinate system $\left(x_{1}, x_{2}, x_{3}\right)$ is introduced such that the plane $x_{3}=0$ coincides with the flat surface of the elastomer, as shown in Fig. 1. The half space is under a gravitational field.

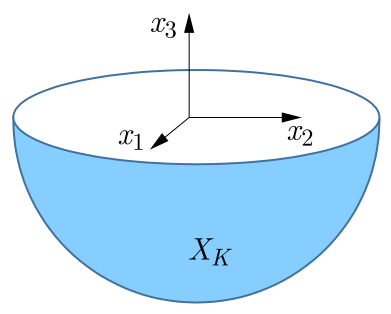

Initial configuration

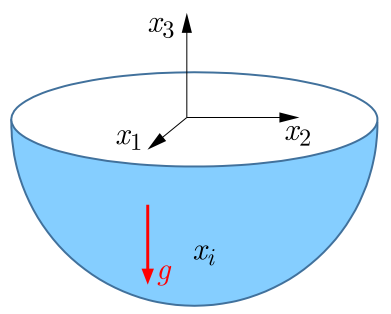

Intermediate configuration

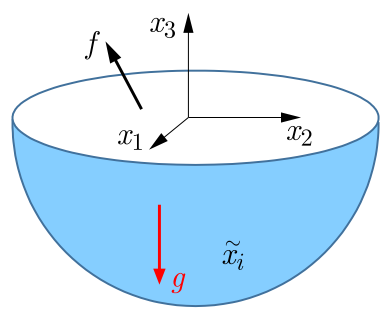

Current configuration

Fig. 1. Schematics of three configurations for the half space elastomer incorporating the gravity effect: initial configuration, intermediate configuration and current configuration. A Cartesian coordinate system $\left(x_{1}, x_{2}, x_{3}\right)$ is introduced in three configurations, where the plane $x_{3}=0$ coincides with the flat surface of the soft elastomer in the intermediate configuration, and the $x_{1^{-}}$and $x_{2}$-axes are along the directions of $x_{1}$ and $x_{2}$, respectively. Referring to this coordinate system, the position vectors of a representative point in the three configurations are represented by $X_{K}, x_{i}\left(X_{K}\right), \widetilde{x}_{i}\left(X_{K}\right)$, respectively

The kinematics is described by the deformation gradient defined as

$$
\bar{F}_{i K}=\frac{\partial x_{i}}{\partial X_{K}}
$$

A concentrated force $f_{i}$ is applied at $x_{i}$ on the surface $x_{3}=0$ in the intermediate state, and the spatial position $x_{i}$ is perturbed with infinitesimal deformation $u_{i}$. In this circumstance, the additional strains induced by the external concentrated force $f_{i}$ are small, and the incremental displacement field $u_{i}(x)$ in the elastomer resulted from any distributed loading can be obtained 
by linear superposition. The spatial coordinate $\widetilde{x}_{i}$ in the current state due to perturbation equals to

$$
\widetilde{x}_{i}=x_{i}+u_{i}
$$

The deformation gradient in the current state is

$$
F_{i K}=\frac{\partial \widetilde{x}_{i}}{\partial X_{K}}=\left(\delta_{i j}+u_{i, j}\right) \bar{F}_{j K}
$$

where $\delta_{i j}$ is the Kronecker delta and $u_{i, j}=\partial u_{i} / \partial x_{j}$. For simplicity, we assume that the material of the elastomer is described by a neo-Hookean constitutive model which takes the strain energy function $W\left(F_{i K}\right)$ as follows (Treloar, 1975)

$$
W=\frac{\mu}{2}\left(F_{i K} F_{i K}-3\right)-p^{*}(J-1)
$$

where $J$ is the determinant of the deformation gradient $F_{i K}, \mu$ is the shear modulus, and $p^{*}$ (interpreted as a pressure) is a Lagrange multiplier concerned with the incompressibility constraint $J-1=0$. The first Piola-Kirchhoff stress $S_{i K}$ is calculated from the stress-strain relation

$$
S_{i K}=\frac{\partial W}{\partial F_{i K}}=\mu F_{i K}-p^{*} F_{K i}^{-1}
$$

In the presence of body forces, the nominal stress in the initial state satisfies the following force balance equation and boundary condition

$$
\begin{aligned}
& S_{i K, K}+\rho g_{i}=0 \\
& S_{i K} N_{K} d A=f_{i} \delta\left(x_{1}\right)\left(x_{2}\right) d a \quad \text { at } \quad x_{3}=0
\end{aligned}
$$

where $d A$ and $N_{K}$ are the element of area and the unit outward normal vector in the initial state, respectively, $d a$ is the element of area in the intermediate state and $\delta(\cdot)$ is the Dirac function.

For convenience, we take the intermediate state as the reference state, and rewrite the force balance equations, and boundary condition with application of Nanson's formula $N_{K} d A=\bar{F}_{i K} n_{i} d a$

$$
\begin{aligned}
& \left(S_{i K} \bar{F}_{j K}\right)_{, j}=0 \\
& S_{i K} \bar{F}_{j K} n_{j}=f_{i} \delta\left(x_{1}\right)\left(x_{2}\right) \quad \text { at } \quad x_{3}=0 \\
& u_{i}=0 \quad \text { at } \quad x_{3}=-\infty
\end{aligned}
$$

where $n_{j}$ is the unit outward normal vector in the reference state.

Since the perturbed displacement is small, all the involved equations can be linearized. For this sake, one can separate the pressure $p^{*}$ into the form $p^{*}=\bar{p}+p$, where $\bar{p}$ is linearly dependent hydrostatic pressure which can be determined as $\bar{p}=\mu-\rho g x_{3}$ in terms of boundary condition $(2.7)_{2}$ (where $f_{i}=0$ ) and $p$ is the incremental pressure from the reference state to the current state. With the linear terms remained only, the incompressibility condition can result in

$$
u_{i, i}=0
$$

For convenience of the following calculation, $S_{i K} \bar{F}_{j K}$ in Eqs. $(2.7)_{1}$ and $(2.7)_{2}$ is expressed as below

$$
S_{i K} \bar{F}_{j K}=-p \delta_{i j}+\mu\left(u_{i, j}+u_{j, i}\right)+\rho g x_{3}\left(\delta_{i j}-u_{j, i}\right)
$$


By the aid of formula (2.9), the linearized forms of Eqs. $(2.7)_{1}$ and $(2.7)_{2}$ are derived as

$$
\begin{aligned}
& \mu u_{i, j j}-p_{, i}-\rho g u_{3, i}=0 \\
& -p \delta_{i j} n_{j}+\mu\left(u_{i, j}+u_{j, i}\right) n_{j}=f_{i} \delta\left(x_{1}\right)\left(x_{2}\right) \quad \text { at } \quad x_{3}=0 \\
& u_{i}=0 \quad \text { at } \quad x_{3}=-\infty
\end{aligned}
$$

Equations (2.10) entirely depict the boundary value problem for the incremental deformation induced by a force exerted on the surface of an elastomer occupying the half space.

For convenience, we rewrite Eqs. $(2.10)_{1}$ and $(2.10)_{2}$ in a more explicit form

$$
\begin{array}{ll}
\mu\left(u_{i, 11}+u_{i, 22}+u_{i, 33}\right)-p_{, i}-\rho g u_{3, i}=0 \\
\mu\left(u_{1,3}+u_{3,1}\right)=f_{1} \delta\left(x_{1}\right)\left(x_{2}\right) \quad & \mu\left(u_{2,3}+u_{3,2}\right)=f_{2} \delta\left(x_{1}\right)\left(x_{2}\right) \\
2 \mu u_{3,3}-p=f_{3} \delta\left(x_{1}\right)\left(x_{2}\right) \quad \text { at } & x_{3}=0
\end{array}
$$

In view of the incompressibility of the material, we adopt two stream functions $\varphi_{1}=\varphi_{1}\left(x_{i}\right)$ and $\varphi_{2}=\varphi_{2}\left(x_{i}\right)$ such that the incremental displacement can be expressed as below

$$
u_{1}=\varphi_{1,3} \quad u_{2}=\varphi_{2,3} \quad u_{3}=-\varphi_{1,1}-\varphi_{2,2}
$$

Substituting Eq. (2.12) into Eq. (2.11) $)_{1}$ and taking the corresponding Fourier transforms in the $x_{1}$ and $x_{2}$ directions, we have

$$
\begin{aligned}
& \mu \Phi_{1,333}-\mu \mathbf{k}^{2} \Phi_{1,3}+\mathrm{i} k_{1} P-k_{1} \rho g\left(k_{1} \Phi_{1}+k_{2} \Phi_{2}\right)=0 \\
& \mu \Phi_{2,333}-\mu \mathbf{k}^{2} \Phi_{2,3}+\mathrm{i} k_{2} P-k_{2} \rho g\left(k_{1} \Phi_{1}+k_{2} \Phi_{2}\right)=0 \\
& \mu\left(k_{1} \Phi_{1}+k_{2} \Phi_{2}\right)_{, 33}-\mu \mathbf{k}^{2}\left(k_{1} \Phi_{1}+k_{2} \Phi_{2}\right)+\mathrm{i} P_{, 3}-\rho g\left(k_{1} \Phi_{1}+k_{2} \Phi_{2}\right)_{, 3}=0
\end{aligned}
$$

where $\mathrm{i}=\sqrt{-1}, \mathbf{k}$ is a two-dimensional vector with components $k_{1}$ and $k_{2}, \Phi_{i}$ and $P$ represented by Eq. $(2.14)_{2}$ as below, are the Fourier transforms of $\varphi_{i}$ and $p$

$$
\begin{aligned}
\Phi_{i}\left(k_{1}, k_{2}, x_{3}\right) & =\int_{-\infty}^{\infty} \int_{-\infty}^{\infty} \varphi_{i}\left(x_{1}, x_{2}, x_{3}\right) \mathrm{e}^{\mathrm{i} \mathbf{k x}} d x_{1} d x_{2} \\
P\left(k_{1}, k_{2}, x_{3}\right) & =\int_{-\infty}^{\infty} \int_{-\infty}^{\infty} p\left(x_{1}, x_{2}, x_{3}\right) \mathrm{e}^{\mathrm{i} \mathbf{k x}} d x_{1} d x_{2}
\end{aligned}
$$

The corresponding boundary conditions are given at $x_{3}=0$

$$
\begin{array}{ll}
\mu \Phi_{1,33}+\mu k_{1}\left(k_{1} \Phi_{1}+k_{2} \Phi_{2}\right)=f_{1} & \mu \Phi_{2,33}+\mu k_{2}\left(k_{1} \Phi_{1}+k_{2} \Phi_{2}\right)=f_{2} \\
2 \mathrm{i} \mu\left(k_{1} \Phi_{1}+k_{2} \Phi_{2}\right)_{, 3}-P=f_{3} &
\end{array}
$$

and at $x_{3}=-\infty$

$$
\Phi_{1,3}=0 \quad \Phi_{2,3}=0 \quad k_{1} \Phi_{1}+k_{2} \Phi_{2}=0
$$

After a series of operations, the general solutions to Eqs. (2.13) can be obtained

$$
\begin{aligned}
& \Phi_{1}=\left(a_{1}+a_{2} k_{1} x_{3}\right) \mathrm{e}^{k x_{3}} \quad \Phi_{2}=\left(a_{3}+a_{2} k_{2} x_{3}\right) \mathrm{e}^{k x_{3}} \\
& P=\mathrm{i}\left[-\left(a_{1} k_{1}+a_{3} k_{2}\right) \rho g+a_{2} k^{2}\left(2 \mu-\rho g x_{3}\right)\right] \mathrm{e}^{k x_{3}}
\end{aligned}
$$


where $k=\sqrt{k_{1}^{2}+k_{2}^{2}}, a_{1}, a_{2}, a_{3}$ can be solved as follows

$$
\begin{aligned}
& a_{1}=\frac{-\mathrm{i} k^{2} k_{1} \mu f_{3}+k_{2}\left(f_{1} k_{2}-f_{2} k_{1}\right)(\rho g+2 k \mu)}{\mu k^{4}(\rho g+2 k \mu)} \\
& a_{2}=\frac{2 \mathrm{i} k^{2} \mu f_{3}+\left(f_{1} k_{1}+f_{2} k_{2}\right)(\rho g+2 k \mu)}{2 \mu k^{3}(\rho g+2 k \mu)} \\
& a_{3}=\frac{-\mathrm{i} k^{2} k_{2} \mu f_{3}+k_{1}\left(f_{2} k_{1}-f_{1} k_{2}\right)(\rho g+2 k \mu)}{\mu k^{4}(\rho g+2 k \mu)}
\end{aligned}
$$

By substituting Eqs. (2.17) into Eq. (2.13) $)_{2}$, one obtains Fourier transforms of the incremental displacements of the surface of the half space

$$
\begin{aligned}
& U_{1}\left(x_{3}=0\right)=\Phi_{1,3}=\left(a_{1} k+a_{2} k_{1}\right) \quad U_{2}\left(x_{3}=0\right)=\Phi_{2,3}=\left(a_{3} k+a_{2} k_{2}\right) \\
& U_{3}\left(x_{3}=0\right)=\mathrm{i} k_{1} \Phi_{1}+\mathrm{i} k_{2} \Phi_{2}=\mathrm{i}\left(a_{1} k_{1}+a_{3} k_{2}\right)
\end{aligned}
$$

The results in Eqs. (2.19) enable the surface incremental displacements of the half space to be solved. We write out the incremental displacements in the form of $u_{i}(\mathbf{x})=G_{i j}\left(\mathbf{x}-\mathbf{x}^{\prime}\right) f_{j}$ by introducing the surface Green's function $G_{i j}\left(\mathbf{x}-\mathbf{x}^{\prime}\right)$ given by

$$
\begin{aligned}
& G_{11}\left(\mathbf{x}-\mathbf{x}^{\prime}\right)=\frac{1}{4 \pi^{2}} \int_{-\infty}^{\infty} \int_{-\infty}^{\infty} \frac{k^{2}+k_{2}^{2}}{2 k^{3} \mu} \mathrm{e}^{-\mathrm{i} \mathbf{k}\left(\mathbf{x}-\mathbf{x}^{\prime}\right)} d k_{1} d k_{2} \\
& G_{22}\left(\mathbf{x}-\mathbf{x}^{\prime}\right)=\frac{1}{4 \pi^{2}} \int_{-\infty}^{\infty} \int_{-\infty}^{\infty} \frac{k^{2}+k_{1}^{2}}{2 k^{3} \mu} \mathrm{e}^{-i \mathbf{k}\left(\mathbf{x}-\mathbf{x}^{\prime}\right)} d k_{1} d k_{2} \\
& G_{33}\left(\mathbf{x}-\mathbf{x}^{\prime}\right)=\frac{1}{4 \pi^{2}} \int_{-\infty}^{\infty} \int_{-\infty}^{\infty} \frac{1}{\rho g+2 k \mu} \mathrm{e}^{-\mathrm{i} \mathbf{k}\left(\mathbf{x}-\mathbf{x}^{\prime}\right)} d k_{1} d k_{2} \\
& G_{12}\left(\mathbf{x}-\mathbf{x}^{\prime}\right)=G_{21}\left(\mathbf{x}-\mathbf{x}^{\prime}\right)=-\frac{1}{4 \pi^{2}} \int_{-\infty}^{\infty} \int_{-\infty}^{\infty} \frac{k_{1} k_{2}}{2 k^{3} \mu} \mathrm{e}^{-i \mathbf{k}\left(\mathbf{x}-\mathbf{x}^{\prime}\right)} d k_{1} d k_{2} \\
& G_{13}\left(\mathbf{x}-\mathbf{x}^{\prime}\right)=G_{31}\left(\mathbf{x}-\mathbf{x}^{\prime}\right)=0
\end{aligned}
$$

Equation $(2.20)_{3}$ can be degenerated to the state of no gravity

$$
G_{33}\left(\mathbf{x}-\mathbf{x}^{\prime}\right)=\frac{1}{4 \pi^{2}} \int_{-\infty}^{\infty} \int_{-\infty}^{\infty} \frac{1}{2 k \mu} \mathrm{e}^{-\mathrm{i} \mathbf{k}\left(\mathbf{x}-\mathbf{x}^{\prime}\right)} d k_{1} d k_{2}
$$

which is in agreement with the result in (He, 2008). After integration, the analytical forms of the Green's function of the surface of the half space can be provided as below

$$
\begin{aligned}
& G_{11}\left(\mathbf{x}-\mathbf{x}^{\prime}\right)=\frac{1}{2 \pi \mu r}\left(1-\frac{\left(x_{2}-x_{2}^{\prime}\right)^{2}}{2 r^{2}}\right) \quad G_{22}\left(\mathbf{x}-\mathbf{x}^{\prime}\right)=\frac{1}{2 \pi \mu r}\left(1-\frac{\left(x_{1}-x_{1}^{\prime}\right)^{2}}{2 r^{2}}\right) \\
& G_{33}\left(\mathbf{x}-\mathbf{x}^{\prime}\right)=\frac{1}{4 \pi \mu r}\left\{1-m r\left[m r_{1} F_{2}\left(1 ; \frac{3}{2}, \frac{3}{2} ;-\frac{m^{2} r^{2}}{4}\right)-\frac{\pi}{2} Y_{0}(m r)\right]\right\} \\
& G_{12}\left(\mathbf{x}-\mathbf{x}^{\prime}\right)=G_{21}\left(\mathbf{x}-\mathbf{x}^{\prime}\right)=\frac{\left(x_{1}-x_{1}^{\prime}\right)\left(x_{2}-x_{2}^{\prime}\right)}{4 \pi \mu r^{3}}
\end{aligned}
$$

where $m$ is defined as $\rho g /(2 \mu), r=\left|\mathbf{x}-\mathbf{x}^{\prime}\right|, F_{j}\left(a_{1}, \ldots, a_{i} ; b_{1}, \ldots, b_{j} ; x\right)$ is the generalized hypergeometric function, and $Y_{0}(x)$ is the Bessel function of the second kind with the order zero. 


\section{Elastic deformation caused by uniform distributed pressure on the surface of the elastomer incorporating the gravity effect}

In this Section, we will apply the above derived surface Green's function to calculate the elastic deformation resulted from uniform distributed pressure. A uniform distributed pressure $q\left(\mathbf{x}^{\prime}\right)$ with loading circular radius $R$ is exerted on the surface of the elastomer occupying a half space, so the surface normal stress is $-q$ in the loading region while the surface normal stress is zero outside the loading region.

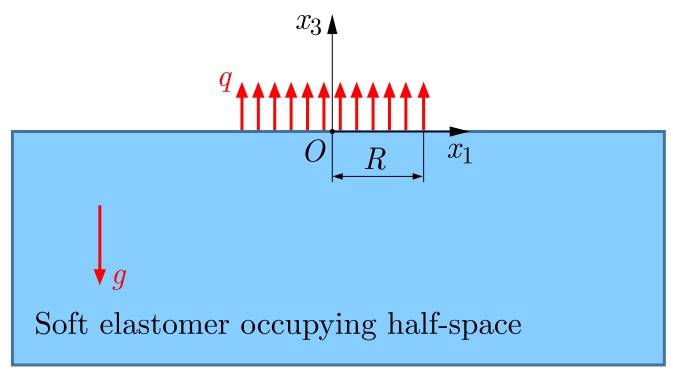

Fig. 2. Schematics of a uniform distributed pressure applied on the surface of a soft elastomer occupying a half space. The uniform distributed pressure $q$ is exerted on a circular zone with radius $R$ of the surface of the elastomer occupying the half space. Density of the soft elastomer is $\rho$, and gravitational acceleration is $g$

The normal surface displacement $u_{3}(\mathbf{x})$ resulted from the pressure can be gained by the integration form

$$
u_{3}(\mathbf{x})=\iint_{A} G_{33}\left(\mathbf{x}-\mathbf{x}^{\prime}\right) q\left(\mathbf{x}^{\prime}\right) d x_{1}^{\prime} d x_{2}^{\prime}
$$

where $A$ is the loading area.

Taking advantage of the polar coordinate transformation as below

$$
x_{1}^{\prime}=x_{1}+r \cos \theta \quad x_{2}^{\prime}=x_{2}+r \sin \theta
$$

Thus, Eq. (3.1) can be adapted by the strategy (Zheng, 2017) as

$$
u_{3}(\mathbf{x})=\int_{0}^{\pi} \int_{r_{1}}^{r_{2}} G_{33}(r, \theta) q(r, \theta) r d r d \theta
$$

where the integration limits $r_{1}$ and $r_{2}$ are determined by the following solution to the boundary equation

$$
\left(x_{1}+r_{1,2} \cos \theta\right)^{2}+\left(x_{2}+r_{1,2} \sin \theta\right)^{2}=R^{2}
$$

According to Eq. (3.4), the two limits of $r$ are on the edge of the circular loading area as

$$
r_{1,2}=-\left(x_{1} \cos \theta+x_{2} \sin \theta\right) \pm \sqrt{R^{2}-\left(x_{1} \sin \theta-x_{2} \cos \theta\right)^{2}}
$$

Substituting Eq. (2.22) 3 into Eq. (3.3) can give a rise to

$$
u_{3}(\mathbf{x})=\frac{q}{4 \pi \mu} \int_{0}^{2 \pi} d \theta \int_{0}^{r_{2}}\left\{1-m r\left[m r_{1} F_{2}\left(1 ; \frac{3}{2}, \frac{3}{2} ;-\frac{m^{2} r^{2}}{4}\right)-\frac{\pi}{2} Y_{0}(m r)\right]\right\} d r=\frac{q}{4 \pi \mu} I_{1}
$$


where $I_{1}$ is an integral with respect to $\theta$ as below, which needs numerical computation

$$
\begin{aligned}
I_{1}= & \int_{0}^{2 \pi}\left\{\frac { 1 } { m } \pi \text { MeijerG } \left[\left\{\{1\},\left\{\frac{1}{2}\right\} ;\left\{\{1,1\},\left\{0, \frac{1}{2}\right\}\right\} ; \frac{1}{4} m^{2} r_{2}^{2}\right]\right.\right. \\
& \left.+\frac{1}{2} r_{2}\left[2-\frac{2}{3} m^{2} r_{2}^{2} F_{2}\left(1 ; \frac{3}{2}, \frac{5}{2} ;-\frac{m^{2} r_{2}^{2}}{4}\right)\right]\right\} d \theta
\end{aligned}
$$

where MeijerG $\left[\left\{\left\{a_{1}, \ldots, a_{n}\right\},\left\{a_{n+1}, \ldots, a_{p}\right\}\right\} ;\left\{\left\{b_{1}, \ldots, b_{m}\right\},\left\{b_{m+1}, \ldots, b_{q}\right\}\right\} ; z\right]$ is the MeijerG function.

By numerically calculating Eqs. (3.6) and (3.7) in Matlab software, we can obtain the normal surface displacement of the soft elastomer driven by the uniform distributed pressure. Since the surface normal displacement caused by the pressure on the elastomer occupying the half space is axisymmetric, we will just plot the normalized surface displacement along the radial direction. Figure 3 presents a 3D surface profile inside $(r / R<1)$ on the boundary $(r / R=1)$ and outside $(1<r / R)$ the loading zone with variation of dimensionless gravity parameter.

(a)

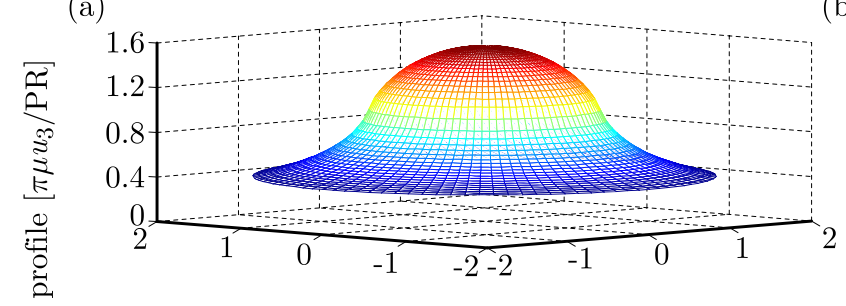

(c)

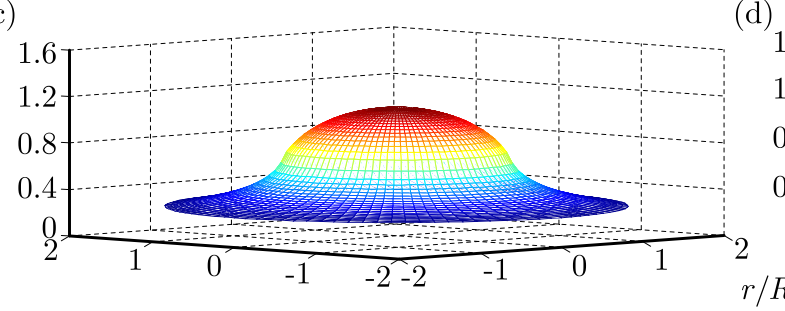

(b)

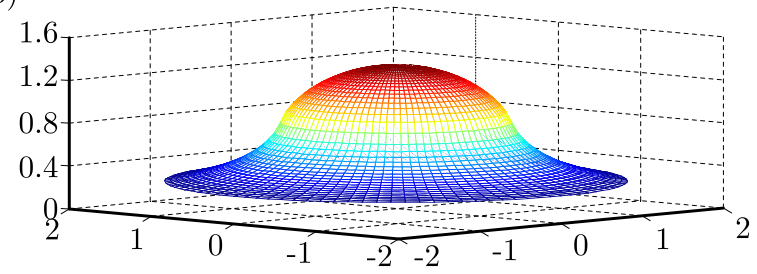

(d)

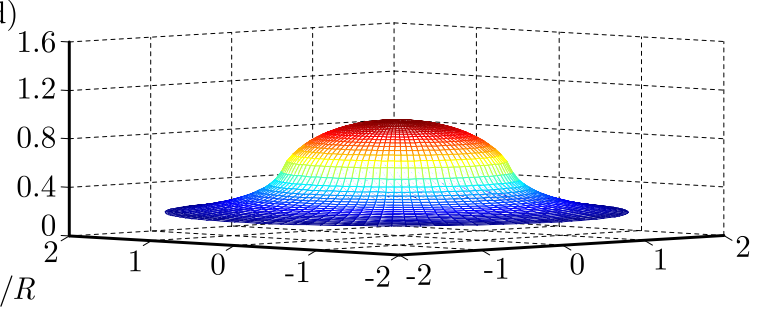

Fig. 3. The 3D surface profile inside $(r / R<1)$ on the boundary $(r / R=1)$ and outside $(1<r / R)$ the loading zone with variation of the dimensionless gravity parameter. With an increase of the gravity parameter, the amplitude of surface deformation decreases

Figure 4 plots the normal surface displacement of the soft elastomer in the loading zone for different dimensionless gravity parameters. The results show that the gravitational force of the soft elastomer has a tremendous impact on the surface deformation of the elastomer. When the normalized gravity parameter $\rho g R / \mu$ is small, i.e., the gravity effect is trivial, the computed normal displacement by the derived Green's function is nearly the same as the well-known analytical solution (Ling, 2002). With an increase of the dimensionless gravity parameter $\rho g R / \mu$, the normal surface displacement in the loading zone decreases. Some biological tissues are commonly soft with Young's modulus ranging from several hundreds to several thousands $\mathrm{Pa}$ (Du et al., 2011). In these cases, the gravity effect on the deformation of soft tissues should be considered.

From the displacement distribution of the elastomer in the loading zone, as shown in Fig. 4, it is found that the maximum normal surface displacement appears at the center of the loading zone, which is the same as in the results reported by Li and Cai (2014). Figure 5 plots the normal surface displacement at the center of the loading zone as a function of the dimensionless gravity parameters. The result shows that the normal surface displacement drastically decreases with an increase of the gravity parameters. 


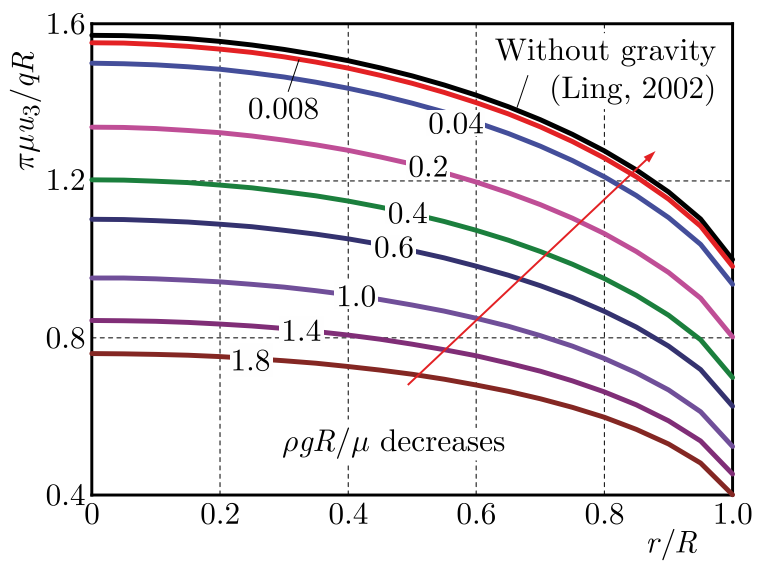

Fig. 4. The axisymmetric normal surface displacement along the radial direction caused by a uniform distributed pressure on the elastomer occupying a half space, for different gravity parameters. The results show that the normal surface displacement in the loading zone decreases with the increasing distance from the center of the loading zone. When the gravity effect is trivial, the normal surface displacement computed by the derived Green's function is nearly the same as the well-known analytical solution (Ling, 2002)

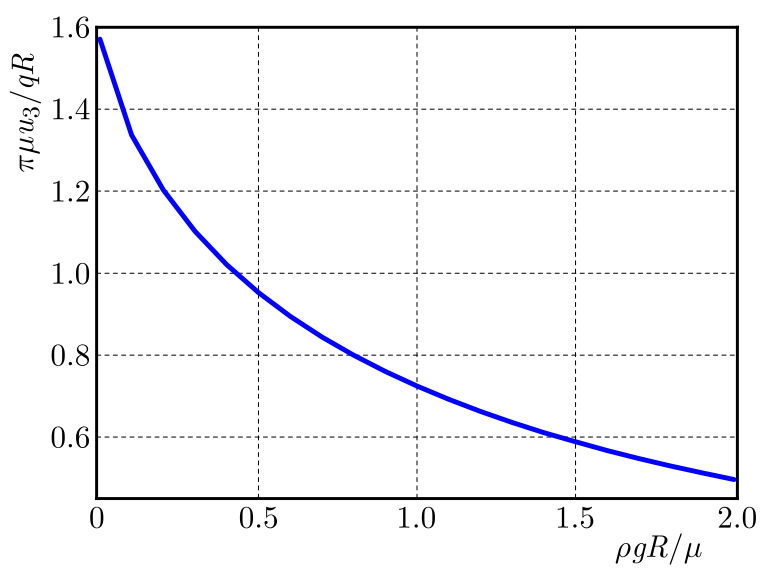

Fig. 5. The dimensionless normal surface displacement at the center of the loading zone as a function of the dimensionless gravity parameter $\rho g R / \mu=0$ corresponds to the circumstance without the gravity effect. The result shows that the surface normal displacement decreases with an increase of the gravity parameter, and the gravitational force prefers to resist the surface deformation, which is consistent with the intuition

In this work, thickness of the soft elastomer is assumed to be infinite, and the influence of thickness of the elastomer is not considered. The previous literature shows that thickness of the soft elastomer has also an important impact on the surface displacement on thin soft layers (Mora et al., 2014; Liang and Cai, 2015). In the future work, we will further explore the surface elastic deformation of soft elastomer layers with a finite thickness and the gravity effect. In the current work, we focus on the gravity effect on the surface deformation of an elastomer. However, for a soft elastomer, the surface stress of the elastomer may also have a big impact on the deformation of the elastomer (He and Lim, 2006), and should be considered in the analysis of the mechanical phenomenon in the future.

\section{Conclusion}

We proposed the surface Green's function of the elastomer occupying a half space based on the neo-Hookean constitutive model, incorporating the gravity effect. By utilizing Fourier transfor- 
mation, the surface Green's function has been analytically derived. We apply the surface Green's function to calculate the surface normal deformation of the soft elastomer and obtain the relation between the surface normal displacement and the gravity parameter. The study shows that gravity can play a tremendous effect on deformation of the soft elastomer resulting from uniform distributed pressure. The proposed surface Green's function has also potential applications in other fields, such as indentation, cell migration, robot walking on soft materials, etc.

\section{Acknowledgements}

X.D. Liang acknowledges the support from the Key Project of of Natural Science Research of Universities in Anhui (Grant No. KJ2018A0558) and the Talent Research Fund Project of Hefei University (Grant No. 16-17RC02). This work is also supported by the doctoral startup foundation from Anhui Jianzhu University (Grant No. 2017QD06) and the Chinese Natural Science Foundation (Grant No. 51608005 and 11402001).

\section{References}

1. Amar M.B., Jia F., 2013, Anisotropic growth shapes intestinal tissues during embryogenesis, Proceedings of the National Academy of Sciences, 110, 26, 10525

2. Biot M.A., 1963, Surface instability of rubber in compression, Applied Scientific Research, Section $A, \mathbf{1 2}, 2,168-182$

3. Віот M.A., 1965, Mechanics of Incremental Deformations: Theory of Elasticity and Viscoelasticity of Initially Stressed Solids and Fluids, Including Thermodynamic Foundations and Applications to Finite Strain, Wiley

4. Dervaux J., Amar M.B., 2012, Mechanical instabilities of gels, Annual Review of Condensed Matter Physics, 3, 311-332

5. Du J., Chen X., Liang X., Zhang G., Xu J., He L., Zhan Q., Feng X.Q., Chien S., Yang C., 2011, Integrin activation and internalization on soft ECM as a mechanism of induction of stem cell differentiation by ECM elasticity, Proceedings of the National Academy of Sciences, 108, 23, 9466-9471

6. Gent A.N., Cho I. S., 1999, Surface instabilities in compressed or bent rubber blocks, Rubber Chemistry and Technology, 72, 2, 253-262

7. He L., 2008, Elastic interaction between force dipoles on a stretchable substrate, Journal of the Mechanics and Physics of Solids, 56, 2957-2971

8. He L.H., Lim C.W., 2006, Surface Green function for a soft elastic half-space: influence of surface stress, International Journal of Solids and Structures, 43, 132-143

9. Keplinger C., Li T., Baumgartner R., Suo Z., Bauer S., 2012, Harnessing snap-through instability in soft dielectrics to achieve giant voltage-triggered deformation, Soft Matter, 8, 285-288

10. Kim J., Hanna J.A., Byun M., Santangelo C.D., Hayward R.C., 2012, Designing responsive buckled surfaces by halftone gel lithography, Science, 335, 1201-1205

11. Li K., CAI S., 2014, Wet adhesion between two soft layers, Soft Matter, 10, 41, 8202-8209

12. Li K., GE D.L., CAI S.Q., 2012, Gravity-induced wrinkling of thin films on soft substrates, Europhysics Letters, 100, 54004

13. Liang X., CAI S., 2015, Gravity induced crease-to-wrinkle transition in soft materials, Applied Physics Letters, 106, 041907

14. Ling F.F., Lai W.M., LucCa D.A., 2002, Fundamentals of Surface Mechanics: With Applications, Springer-Verlag, New York

15. Mora S., Phou T., Fromental J.M., Pomeau Y., 2014, Gravity driven instability in elastic solid layers, Physical Review Letters, 113, 17, 178301 
16. Ogden R.W., 1997, Non-Linear Elastic Deformations, Courier Corporation

17. Tanaka T., Sun S. T., Hirokawa Y., Katayama S., Kucera J., Hirose Y., Amiya T., 1987, Mechanical instability of gels at the phase transition, Nature, 325, 796-798

18. TrelonR L.R.G., 1975, The Physics of Rubber Elasticity, Oxford University Press

19. WAng Q., ZhaO X., 2013, Creasing-wrinkling transition in elastomer films under electric fields, Physical Review E, 88, 4, 042403

20. Zheng Y., Crosby A.J., CAi S., 2017, Indentation of a stretched elastomer, Journal of the Mechanics and Physics of Solids, 107, 145-159

21. Zhu J., Stoyanov H., Kofod G., Suo Z., 2010, Large deformation and electromechanical instability of a dielectric elastomer tube actuator, Journal of Applied Physics, 108, 074113

Manuscript received April 25, 2019; accepted for print June 17, 2019 